\title{
Pairwise Precontinuity in Intuitionistic Smooth Bitopological Spaces
}

Jin Tae Kim and Seok Jong Lee

Department of Mathematics, Chungbuk National University, Cheongju, Korea

\section{]jfis}

\begin{abstract}
The authors introduced the concepts of intuitionistic smooth bitopological spaces, and obtained some characterizations of intuitionistic fuzzy pairwise continuity and intuitionistic fuzzy pairwise semicontinuity of them in the previous papers. Here, we introduce a new notion of intuitionistic fuzzy pairwise precontinuous mappings in intuitionistic smooth bitopological spaces, and obtain six equivalent conditions for intuitionistic fuzzy pairwise precontinuous mappings. Furthermore, we obtain another equivalent condition for a bijective mapping.
\end{abstract}

Keywords: Intuitionistic smooth bitopology, Pairwise preopen, Pairwise preclosed, Pairwise precontinuous

\section{Introduction and Preliminaries}

Chang [1] introduced a new concept called the fuzzy topology in a fuzzy set area. The fuzzy topology is a crisp subfamily of fuzzy sets, and hence, it does not represent the notion of vagueness of a fuzzy set. This would be a shortcoming in the process of fuzzification of topological spaces. To overcome these defects, a new definition of fuzzy topology using the openness was introduced by Šostak [2, 3]. His fuzzy topology was later rephrased as "smooth topology" by Ramadan [4].

Also, Chang's fuzzy topological spaces were generalized into the concept of fuzzy bitopological spaces with pairing the fuzzy topological spaces by Kandil [5]. His fuzzy bitopological spaces were later generalized into a notion of smooth bitopological spaces by Lee and his colleagues [6, 7] by adding vagueness.

On the other hand, the intuitionistic fuzzy topological spaces were introduced by Çoker and

Received: Jul. 7, 2019

Revised : Aug. 19, 2019

Accepted: Sep. 20, 2019

Correspondence to: Seok Jong Lee (sjl@cbnu.ac.kr)

(CThe Korean Institute of Intelligent Systems

(c) This is an Open Access article distributed under the terms of the Creative Commons Attribution Non-Commercial License (http://creativecommons.org/licenses/ by-nc/3.0// which permits unrestricted noncommercial use, distribution, and reproduction in any medium, provided the original work is properly cited. his colleague [8, 9]. They are defined with intuitionistic fuzzy sets introduced by Atanassov [10]. Also, new generalization of a smooth topology was introduced by Mondal and Samanta [11], which is called intuitionistic gradation of openness. Lim et al. [12] defined the term "intuitionistic smooth topology," which is a slight modification of the Mondal and Samanta's intuitionistic gradation of openness. Thus, it is different from ours.

In the previous papers [13, 14], we introduced the concepts of intuitionistic smooth bitopological spaces, and obtained some characterizations of intuitionistic fuzzy pairwise continuity and intuitionistic fuzzy pairwise semicontinuity of them.

In this paper, by using the idea of intuitionistic fuzzy pairwise preopen and preclosed sets, we introduce intuitionistic fuzzy pairwise precontinuous mappings in intuitionistic smooth bitopological spaces, and obtain the characterizations for the mappings. 
Throughout this paper, $I$ denotes the unit interval $[0,1]$ of the real line and $I_{0}=(0,1]$. A member $\mu$ of $I^{X}$ is called a fuzzy set in $X$. For any $\mu \in I^{X}, \mu^{c}$ denotes the complement $1-\mu$. By $\tilde{0}$ and $\tilde{1}$ we denote constant mappings on $X$ with value of 0 and 1 , respectively.

Let $X$ be a nonempty set. An intuitionistic fuzzy set $A$ is an ordered pair

$$
A=\left(\mu_{A}, \gamma_{A}\right) \text {, }
$$

where the functions $\mu_{A}: X \rightarrow I$ and $\gamma_{A}: X \rightarrow I$ denote the degree of membership and the degree of nonmembership, respectively, and $\mu_{A}+\gamma_{A} \leq 1$. Obviously, every fuzzy set $\mu$ in $X$ is an intuitionistic fuzzy set of the form $(\mu, \tilde{1}-\mu) . I(X)$ denotes a family of all intuitionistic fuzzy sets in $X$ and "IF" stands for intuitionistic fuzzy.

All the definitions and notations which are not mentioned in this paper, we refer to [13, 14].

Definition 1.1 ([13]). An intuitionistic smooth topology on $X$ is a mapping $\mathcal{T}: I(X) \rightarrow I$ which satisfies the following properties:

1) $\mathcal{T}(\underline{0})=\mathcal{T}(\underline{1})=1$.

2) $\mathcal{T}(A \cap B) \geq \mathcal{T}(A) \wedge \mathcal{T}(B)$.

3) $\mathcal{T}\left(\bigvee A_{i}\right) \geq \wedge \mathcal{T}\left(A_{i}\right)$

The pair $(X, \mathcal{T})$ is called an intuitionistic smooth topological space.

Definition 1.2 ( [13]). A system $\left(X, \mathcal{T}_{1}, \mathcal{T}_{2}\right)$ consisting of a set $X$ with two intuitionistic smooth topologies $\mathcal{T}_{1}$ and $\mathcal{T}_{2}$ on $X$ is called a intuitionistic smooth bitopological space(ISBTS for short). Throughout this paper the indices $i, j$ take the value in $\{1,2\}$ and $i \neq j$.

Definition 1.3 ( [13]). Let $f:\left(X, \mathcal{T}_{1}, \mathcal{T}_{2}\right) \rightarrow\left(Y, \mathcal{U}_{1}, \mathcal{U}_{2}\right)$ be a mapping from an ISBTS $X$ to an ISBTS $Y$ and $r, s \in I_{0}$. Then $f$ is said to be IF pairwise $(r, s)$-continuous if the induced mapping $f:\left(X, \mathcal{T}_{1}\right) \rightarrow\left(Y, \mathcal{U}_{1}\right)$ is an IF $r$-continuous mapping and the induced mapping $f:\left(X, \mathcal{T}_{2}\right) \rightarrow\left(Y, \mathcal{U}_{2}\right)$ is an IF $s$ continuous mapping.

Definition 1.4 ( [13]). Let $f:\left(X, \mathcal{T}_{1}, \mathcal{T}_{2}\right) \rightarrow\left(Y, \mathcal{U}_{1}, \mathcal{U}_{2}\right)$ be a mapping from an ISBTS $X$ to an ISBTS $Y$ and $r, s \in I_{0}$. Then $f$ is said to be IF pairwise $(r, s)$-semicontinuous if $f^{-1}(A)$ is an IF $\left(\mathcal{T}_{1}, \mathcal{T}_{2}\right)-(r, s)$-semiopen set in $X$ for each IF $\mathcal{U}_{1}$-r $r$-open set $A$ in $Y$ and $f^{-1}(B)$ is an $\operatorname{IF}\left(\mathcal{T}_{2}, \mathcal{T}_{1}\right)-(s, r)$-semiopen set in $X$ for each IF $\mathcal{U}_{2}$-s-open set $B$ in $Y$.
Definition $1.5([14])$. Let $f:\left(X, \mathcal{T}_{1}, \mathcal{T}_{2}\right) \rightarrow\left(Y, \mathcal{U}_{1}, \mathcal{U}_{2}\right)$ be a mapping from an ISBTS $X$ to an ISBTS $Y$ and $r, s \in I_{0}$. Then $f$ is said to be

1) IF pairwise $(r, s)$-open if the induced mapping $f:\left(X, \mathcal{T}_{1}\right)$ $\rightarrow\left(Y, \mathcal{U}_{1}\right)$ is an IF $r$-open mapping and the induced mapping $f:\left(X, \mathcal{T}_{2}\right) \rightarrow\left(Y, \mathcal{U}_{2}\right)$ is an IF $s$-open mapping,

2) IF pairwise $(r, s)$-closed if the induced mapping $f:\left(X, \mathcal{T}_{1}\right)$ $\rightarrow\left(Y, \mathcal{U}_{1}\right)$ is an IF $r$-closed mapping and the induced mapping $f:\left(X, \mathcal{T}_{2}\right) \rightarrow\left(Y, \mathcal{U}_{2}\right)$ is an IF $s$-closed mapping.

Definition 1.6 ([14]). Let $f:\left(X, \mathcal{T}_{1}, \mathcal{T}_{2}\right) \rightarrow\left(Y, \mathcal{U}_{1}, \mathcal{U}_{2}\right)$ be a mapping from an ISBTS $X$ to an ISBTS $Y$ and $r, s \in I_{0}$. Then $f$ is said to be

1) IF pairwise $(r, s)$-semiopen if $f(A)$ is $\operatorname{IF}\left(\mathcal{U}_{1}, \mathcal{U}_{2}\right)-(r, s)$ semiopen in $Y$ for each IF $\mathcal{T}_{1}$-r -open set $A$ in $X$ and $f(B)$ is IF $\left(\mathcal{U}_{2}, \mathcal{U}_{1}\right)-(s, r)$-semiopen in $Y$ for each IF $\mathcal{T}_{2}$-s-open set $B$ in $X$,

2) IF pairwise $(r, s)$-semiclosed if $f(A)$ is $\operatorname{IF}\left(\mathcal{U}_{1}, \mathcal{U}_{2}\right)-(r, s)$ semiclosed in $Y$ for each IF $\mathcal{T}_{1}-r$-closed set $A$ in $X$ and $f(B)$ is $\operatorname{IF}\left(\mathcal{U}_{2}, \mathcal{U}_{1}\right)-(s, r)$-semiclosed in $Y$ for each IF $\mathcal{T}_{2}$ $s$-closed set $B$ in $X$.

\section{Intuitionistic Fuzzy $(r, s)$-preopen and Preclosed Sets}

Now, we define the concepts of intuitionistic fuzzy $\left(\mathcal{T}_{i}, \mathcal{T}_{j}\right)$ $(r, s)$-preopen and preclosed sets, and investigate some of their properties.

Definition 2.1. Let $A$ be an intuitionistic fuzzy set in an ISBTS $\left(X, \mathcal{T}_{1}, \mathcal{T}_{2}\right)$ and $r, s \in I_{0}$. Then $A$ is said to be

1) IF $\left(\mathcal{T}_{i}, \mathcal{T}_{j}\right)-(r, s)$-preopen if $A \subseteq \mathcal{T}_{i}-\operatorname{int}\left(\mathcal{T}_{j}-\mathrm{cl}(A, s), r\right)$,

2) IF $\left(\mathcal{T}_{i}, \mathcal{T}_{j}\right)-(r, s)$-preclosed if $\mathcal{T}_{i}-\operatorname{cl}\left(\mathcal{T}_{j}-\operatorname{int}(A, s), r\right) \subseteq$ A.

Theorem 2.2. Let $A$ be an intuitionistic fuzzy set in an ISBTS $\left(X, \mathcal{T}_{1}, \mathcal{T}_{2}\right)$ and $r, s \in I_{0}$. Then the following statements are equivalent:

1) $A$ is an $\operatorname{IF}\left(\mathcal{T}_{i}, \mathcal{T}_{j}\right)-(r, s)$-preopen set.

2) $A^{c}$ is an $\operatorname{IF}\left(\mathcal{T}_{i}, \mathcal{T}_{j}\right)-(r, s)$-preclosed set.

Proof. 1) $\Rightarrow 2)$ Let $A$ be an $\operatorname{IF}\left(\mathcal{T}_{i}, \mathcal{T}_{j}\right)-(r, s)$-preopen set. Then

$$
A \subseteq \mathcal{T}_{i}-\operatorname{int}\left(\mathcal{T}_{j}-\operatorname{cl}(A, s), r\right) .
$$


Thus, we have

$$
\mathcal{T}_{i}-\operatorname{cl}\left(\mathcal{T}_{j}-\operatorname{int}\left(A^{c}, s\right), r\right)=\mathcal{T}_{i}-\operatorname{int}\left(\mathcal{T}_{j}-\operatorname{cl}(A, s), r\right)^{c} \subseteq A^{c}
$$

Hence, $A^{c}$ is an $\operatorname{IF}\left(\mathcal{T}_{i}, \mathcal{T}_{j}\right)-(r, s)$-preopen set.

$2) \Rightarrow 1)$ Let $A^{c}$ be an $\operatorname{IF}\left(\mathcal{T}_{i}, \mathcal{T}_{j}\right)-(r, s)$-preclosed set. Then

$$
\mathcal{T}_{i}-\operatorname{cl}\left(\mathcal{T}_{j}-\operatorname{int}\left(A^{c}, s\right), r\right) \subseteq A^{c} .
$$

Hence, we obtain

$$
A \subseteq \mathcal{T}_{i}-\operatorname{cl}\left(\mathcal{T}_{j}-\operatorname{int}\left(A^{c}, s\right), r\right)^{c}=\mathcal{T}_{i}-\operatorname{int}\left(\mathcal{T}_{j}-\operatorname{cl}(A, s), r\right) .
$$

Thus, $A$ is an $\operatorname{IF}\left(\mathcal{T}_{i}, \mathcal{T}_{j}\right)-(r, s)$-preopen set.

Theorem 2.3. Let $A$ be an intuitionistic fuzzy set in an ISBTS $\left(X, \mathcal{T}_{1}, \mathcal{T}_{2}\right)$ and $r, s \in I_{0}$. Then

1) If $A$ is IF $\mathcal{T}_{1}$-r -open in $\left(X, \mathcal{T}_{1}\right)$, then $A$ is an $\operatorname{IF}\left(\mathcal{T}_{1}, \mathcal{T}_{2}\right)$ $(r, s)$-preopen set in $\left(X, \mathcal{T}_{1}, \mathcal{T}_{2}\right)$ for all $s \in I_{0}$.

2) If $A$ is $\operatorname{IF} \mathcal{T}_{2}$-s-open in $\left(X, \mathcal{T}_{2}\right)$, then $A$ is an $\operatorname{IF}\left(\mathcal{T}_{2}, \mathcal{T}_{1}\right)$ $(s, r)$-preopen set in $\left(X, \mathcal{T}_{1}, \mathcal{T}_{2}\right)$ for all $r \in I_{0}$.

Proof. 1) Let $A$ be an IF $\mathcal{T}_{1}$-r-open set in $\left(X, \mathcal{T}_{1}\right)$. Then $A=$ $\mathcal{T}_{1}$-int $(A, r)$. Hence, we obtain

$$
A=\mathcal{T}_{1}-\operatorname{int}(A, r) \subseteq \mathcal{T}_{1}-\operatorname{int}\left(\mathcal{T}_{2}-\operatorname{cl}(A, s), r\right) .
$$

Thus, $A$ is $\operatorname{IF}\left(\mathcal{T}_{1}, \mathcal{T}_{2}\right)-(r, s)$-preopen in $\left(X, \mathcal{T}_{1}, \mathcal{T}_{2}\right)$.

2) Similar to 1).

The following example shows that the converses of the above theorem need not be true.

Example 2.4. Let $X=\{x, y\}$ and let $A_{1}, A_{2}, A_{3}$, and $A_{4}$ be intuitionistic fuzzy sets in $X$ defined as

$$
\begin{array}{ll}
A_{1}(x)=(0.2,0.7), & A_{1}(y)=(0.4,0.5) ; \\
A_{2}(x)=(0.6,0.3), & A_{2}(y)=(0.2,0.7) ; \\
A_{3}(x)=(0.2,0.7), & A_{3}(y)=(0.2,0.7) ;
\end{array}
$$

and

$$
A_{4}(x)=(0.5,0.5), \quad A_{4}(y)=(0.1,0.8) .
$$

Define $\mathcal{T}_{1}: I(X) \rightarrow I$ and $\mathcal{T}_{2}: I(X) \rightarrow I$ by

$$
\mathcal{T}_{1}(A)= \begin{cases}1 & \text { if } A=\underline{0}, \underline{1} \\ \frac{1}{2} & \text { if } A=A_{1} \\ 0 & \text { otherwise }\end{cases}
$$

and

$$
\mathcal{T}_{2}(A)= \begin{cases}1 & \text { if } A=\underline{0}, \underline{1} \\ \frac{1}{3} & \text { if } A=A_{2}, \\ 0 & \text { otherwise. }\end{cases}
$$

Then $\left(\mathcal{T}_{1}, \mathcal{T}_{2}\right)$ is an ISBT on $X$. Since

$$
\begin{aligned}
\mathcal{T}_{1}-\operatorname{int}\left(\mathcal{T}_{2}-\operatorname{cl}\left(A_{3}, \frac{1}{3}\right), \frac{1}{2}\right) & =\mathcal{T}_{1}-\operatorname{int}\left(A_{2}^{c}, \frac{1}{3}\right) \\
& =A_{1} \supseteq A_{3},
\end{aligned}
$$

$A_{3}$ is IF $\left(\mathcal{T}_{1}, \mathcal{T}_{2}\right)-\left(\frac{1}{2}, \frac{1}{3}\right)$-preopen in $\left(X, \mathcal{T}_{1}, \mathcal{T}_{2}\right)$. But $A_{3}$ is not an IF $\mathcal{T}_{1}$ - $\frac{1}{2}$-open set in $\left(X, \mathcal{T}_{1}\right)$. Also we have

$$
\begin{aligned}
\mathcal{T}_{2}-\operatorname{int}\left(\mathcal{T}_{1}-\operatorname{cl}\left(A_{4}, \frac{1}{2}\right), \frac{1}{3}\right) & =\mathcal{T}_{2}-\operatorname{int}\left(A_{1}^{c}, \frac{1}{3}\right) \\
& =A_{2} \supseteq A_{4},
\end{aligned}
$$

Thus, $A_{4}$ is $\operatorname{IF}\left(\mathcal{T}_{2}, \mathcal{T}_{1}\right)-\left(\frac{1}{3}, \frac{1}{2}\right)$-preopen in $\left(X, \mathcal{T}_{1}, \mathcal{T}_{2}\right)$. But $A_{4}$ is not a IF $\mathcal{T}_{2}-\frac{1}{3}$-open in $\left(X, \mathcal{T}_{2}\right)$.

The following example shows that $\operatorname{IF}\left(\mathcal{T}_{i}, \mathcal{T}_{j}\right)-(r, s)$-semiopen sets and $\operatorname{IF}\left(\mathcal{T}_{i}, \mathcal{T}_{j}\right)-(r, s)$-preopen sets are independent notions.

Example 2.5. Let $X=\{x, y\}$ and $A_{1}, A_{2}, A_{3}, A_{4}, A_{5}$, and $A_{6}$ be intuitionistic fuzzy sets in $X$ defined as

$$
\begin{aligned}
& A_{1}(x)=(0.1,0.8), \quad A_{1}(y)=(0.6,0.3) ; \\
& A_{2}(x)=(0.7,0.2), \quad A_{2}(y)=(0.2,0.7) ; \\
& A_{3}(x)=(0,0.9), \quad A_{3}(y)=(0.5,0.4) ; \\
& A_{4}(x)=(0.1,0.8), \quad A_{4}(y)=(0.7,0.3) ; \\
& A_{5}(x)=(0.5,0.5), \quad A_{5}(y)=(0.5,0.4) ;
\end{aligned}
$$

and

$$
A_{6}(x)=(0.8,0.1), \quad A_{6}(y)=(0.2,0.7) .
$$

Define $\mathcal{T}_{1}: I(X) \rightarrow I$ and $\mathcal{T}_{2}: I(X) \rightarrow I$ by

$$
\mathcal{T}_{1}(A)= \begin{cases}1 & \text { if } A=\underline{0}, \underline{1} \\ \frac{1}{2} & \text { if } A=A_{1} \\ 0 & \text { otherwise }\end{cases}
$$


and

$$
\mathcal{T}_{2}(A)= \begin{cases}1 & \text { if } A=\underline{0}, \underline{1}, \\ \frac{1}{3} & \text { if } A=A_{2}, \\ 0 & \text { otherwise. }\end{cases}
$$

Then $\left(\mathcal{T}_{1}, \mathcal{T}_{2}\right)$ is an ISBT on $X$. Note that

$$
\begin{aligned}
& \mathcal{T}_{1}-\operatorname{int}\left(\mathcal{T}_{2}-\operatorname{cl}\left(A_{3}, \frac{1}{3}\right), \frac{1}{2}\right)=\mathcal{T}_{1}-\operatorname{int}\left(A_{2}^{c}, \frac{1}{2}\right)=A_{1} \supseteq A_{3}, \\
& \mathcal{T}_{2}-\operatorname{cl}\left(\mathcal{T}_{1}-\operatorname{int}\left(A_{3}, \frac{1}{2}\right), \frac{1}{3}\right)=\mathcal{T}_{2}-\operatorname{cl}\left(\underline{0}, \frac{1}{3}\right)=\underline{0} \nsupseteq A_{3}, \\
& \mathcal{T}_{2}-\operatorname{cl}\left(\mathcal{T}_{1}-\operatorname{int}\left(A_{4}, \frac{1}{2}\right), \frac{1}{3}\right)=\mathcal{T}_{2}-\operatorname{cl}\left(A_{1}, \frac{1}{3}\right)=A_{2}^{c} \supseteq A_{4}, \\
& \mathcal{T}_{1}-\operatorname{int}\left(\mathcal{T}_{2}-\operatorname{cl}\left(A_{4}, \frac{1}{3}\right), \frac{1}{2}\right)=\mathcal{T}_{1}-\operatorname{int}\left(A_{2}^{c}, \frac{1}{2}\right)=A_{1} \nsupseteq A_{4}, \\
& \mathcal{T}_{2}-\operatorname{int}\left(\mathcal{T}_{1}-\operatorname{cl}\left(A_{5}, \frac{1}{2}\right), \frac{1}{3}\right)=\mathcal{T}_{2}-\operatorname{int}\left(\underline{1}, \frac{1}{3}\right)=\underline{1} \supseteq A_{5}, \\
& \mathcal{T}_{1}-\operatorname{cl}\left(\mathcal{T}_{2}-\operatorname{int}\left(A_{5}, \frac{1}{3}\right), \frac{1}{2}\right)=\mathcal{T}_{1}-\operatorname{cl}\left(\underline{0}, \frac{1}{2}\right)=\underline{0} \nsupseteq A_{5}, \\
& \mathcal{T}_{1}-\operatorname{cl}\left(\mathcal{T}_{2}-\operatorname{int}\left(A_{6}, \frac{1}{3}\right), \frac{1}{2}\right)=\mathcal{T}_{1}-\operatorname{cl}\left(A_{2}, \frac{1}{2}\right)=\underline{1} \supseteq A_{6},
\end{aligned}
$$

and

$$
\mathcal{T}_{2}-\operatorname{int}\left(\mathcal{T}_{1}-\operatorname{cl}\left(A_{6}, \frac{1}{2}\right), \frac{1}{3}\right)=\mathcal{T}_{2}-\operatorname{int}\left(A_{1}^{c}, \frac{1}{3}\right)=A_{2} \nsupseteq A_{6} .
$$

Hence, $A_{3}$ is an $\operatorname{IF}\left(\mathcal{T}_{1}, \mathcal{T}_{2}\right)-\left(\frac{1}{2}, \frac{1}{3}\right)$-preopen set which is not $\operatorname{IF}\left(\mathcal{T}_{1}, \mathcal{T}_{2}\right)-\left(\frac{1}{2}, \frac{1}{3}\right)$-semiopen. Also, $A_{4}$ is an $\operatorname{IF}\left(\mathcal{T}_{1}, \mathcal{T}_{2}\right)-\left(\frac{1}{2}, \frac{1}{3}\right)$ semiopen set which is not IF $\left(\mathcal{T}_{1}, \mathcal{T}_{2}\right)-\left(\frac{1}{2}, \frac{1}{3}\right)$-preopen. The set $A_{5}$ is an $\operatorname{IF}\left(\mathcal{T}_{2}, \mathcal{T}_{1}\right)-\left(\frac{1}{3}, \frac{1}{2}\right)$-preopen set which is not $\operatorname{IF}\left(\mathcal{T}_{2}, \mathcal{T}_{1}\right)$ $\left(\frac{1}{3}, \frac{1}{2}\right)$-semiopen. Also, $A_{6}$ is an $\operatorname{IF}\left(\mathcal{T}_{2}, \mathcal{T}_{1}\right)-\left(\frac{1}{3}, \frac{1}{2}\right)$-semiopen set which is not IF $\left(\mathcal{T}_{2}, \mathcal{T}_{1}\right)-\left(\frac{1}{3}, \frac{1}{2}\right)$-preopen.

Theorem 2.6. Let $\left(X, \mathcal{T}_{1}, \mathcal{T}_{2}\right)$ be an ISBTS and $r, s \in I_{0}$. Then the following statements are true:

1) If $\left\{A_{k}\right\}$ is a family of $\operatorname{IF}\left(\mathcal{T}_{i}, \mathcal{T}_{j}\right)-(r, s)$-preopen sets in $X$, then $\bigcup A_{k}$ is $\operatorname{IF}\left(\mathcal{T}_{i}, \mathcal{T}_{j}\right)-(r, s)$-preopen.

2) If $\left\{A_{k}\right\}$ is a family of $\operatorname{IF}\left(\mathcal{T}_{i}, \mathcal{T}_{j}\right)-(r, s)$-preclosed sets in $X$, then $\bigcap A_{k}$ is IF $\left(\mathcal{T}_{i}, \mathcal{T}_{j}\right)$ - $(r, s)$-preclosed.

Proof. (1) Let $\left\{A_{k}\right\}$ be a collection of $\operatorname{IF}\left(\mathcal{T}_{i}, \mathcal{T}_{j}\right)-(r, s)$-preopen sets in $X$. Then for each $k$,

$$
A_{k} \subseteq \mathcal{T}_{i}-\operatorname{int}\left(\mathcal{T}_{j}-\operatorname{cl}\left(A_{k}, s\right), r\right)
$$

Thus, we have

$$
\begin{aligned}
\bigcup A_{k} & \subseteq \bigcup \mathcal{T}_{i}-\operatorname{int}\left(\mathcal{T}_{j}-\operatorname{cl}\left(A_{k}, s\right), r\right) \\
& \subseteq \mathcal{T}_{i}-\operatorname{int}\left(\mathcal{T}_{j}-\operatorname{cl}\left(\bigcup A_{k}, s\right), r\right)
\end{aligned}
$$

Thus, $\bigcup A_{k}$ is $\operatorname{IF}\left(\mathcal{T}_{i}, \mathcal{T}_{j}\right)-(r, s)$-preopen

2) It follows from (1) using Theorem 2.2

Definition 2.7. Let $\left(X, \mathcal{T}_{1}, \mathcal{T}_{2}\right)$ be an ISBTS and $r, s \in I_{0}$. For each $A \in I(X)$, the $I F\left(\mathcal{T}_{i}, \mathcal{T}_{j}\right)$-(r,s)-preinterior is defined by

$\left(\mathcal{T}_{i}, \mathcal{T}_{j}\right)-\operatorname{pint}(A, r, s)$

$=\bigcup\left\{B \in I(X) \mid B \subseteq A, B\right.$ is $\operatorname{IF}\left(\mathcal{T}_{i}, \mathcal{T}_{j}\right)-(r, s)$-preopen $\}$,

and the $I F\left(\mathcal{T}_{i}, \mathcal{T}_{j}\right)-(r, s)$-preclosure is defined by

$\left(\mathcal{T}_{i}, \mathcal{T}_{j}\right)-\operatorname{pcl}(A, r, s)$

$=\bigcap\left\{B \in I(X) \mid A \subseteq B, B\right.$ is $\operatorname{IF}\left(\mathcal{T}_{i}, \mathcal{T}_{j}\right)$ - $(r, s)$-preclosed $\}$.

Obviously, $\left(\mathcal{T}_{i}, \mathcal{T}_{j}\right)-\operatorname{pcl}(A, r, s)$ is the smallest IF $\left(\mathcal{T}_{i}, \mathcal{T}_{j}\right)$ $(r, s)$-preclosed set which contains $A$, and $\left(\mathcal{T}_{i}, \mathcal{T}_{j}\right)-\operatorname{pint}(A, r, s)$ is the greatest IF $\left(\mathcal{T}_{i}, \mathcal{T}_{j}\right)-(r, s)$-preopen set which is contained in $A$. Clearly, $\left(\mathcal{T}_{i}, \mathcal{T}_{j}\right)-\operatorname{pcl}(A, r, s)=A$ for any $\operatorname{IF}\left(\mathcal{T}_{i}, \mathcal{T}_{j}\right)$ $(r, s)$-preclosed set $A$, and $\left(\mathcal{T}_{i}, \mathcal{T}_{j}\right)-\operatorname{pint}(A, r, s)=A$ for any IF $\left(\mathcal{T}_{i}, \mathcal{T}_{j}\right)$ - $(r, s)$-preopen set $A$.

Moreover, we have

$$
\begin{aligned}
\mathcal{T}_{i}-\operatorname{int}(A, r) & \subseteq\left(\mathcal{T}_{i}, \mathcal{T}_{j}\right)-\operatorname{pint}(A, r, s) \\
& \subseteq A \\
& \subseteq\left(\mathcal{T}_{i}, \mathcal{T}_{j}\right)-\operatorname{pcl}(A, r, s) \\
& \subseteq \mathcal{T}_{i}-\operatorname{cl}(A, r)
\end{aligned}
$$

Also, we have the following results:

1) $\left(\mathcal{T}_{i}, \mathcal{T}_{j}\right)-\operatorname{pcl}(\underline{0}, r, s)=\underline{0},\left(\mathcal{T}_{i}, \mathcal{T}_{j}\right)-\operatorname{pcl}(\underline{1}, r, s)=\underline{1}$.

2) $\left(\mathcal{T}_{i}, \mathcal{T}_{j}\right)-\operatorname{pcl}(A, r, s) \supseteq A$.

3) $\left(\mathcal{T}_{i}, \mathcal{T}_{j}\right)-\operatorname{pcl}(A, r, s) \cup\left(\mathcal{T}_{i}, \mathcal{T}_{j}\right)-\operatorname{pcl}(B, r, s)$ $\subseteq\left(\mathcal{T}_{i}, \mathcal{T}_{j}\right)-\operatorname{pcl}(A \cup B, r, s)$.

4) $\left(\mathcal{T}_{i}, \mathcal{T}_{j}\right)-\operatorname{pcl}\left(\left(\mathcal{T}_{i}, \mathcal{T}_{j}\right)-\operatorname{pcl}(A, r, s), r, s\right)$ $=\left(\mathcal{T}_{i}, \mathcal{T}_{j}\right)-\operatorname{pcl}(A, r, s)$.

5) $\left(\mathcal{T}_{i}, \mathcal{T}_{j}\right)-\operatorname{pint}(\underline{0}, r, s)=\underline{0},\left(\mathcal{T}_{i}, \mathcal{T}_{j}\right)-\operatorname{pint}(\underline{1}, r, s)=\underline{1}$.

6) $\left(\mathcal{T}_{i}, \mathcal{T}_{j}\right)-\operatorname{pint}(A, r, s) \subseteq A$.

7) $\left(\mathcal{T}_{i}, \mathcal{T}_{j}\right)-\operatorname{pint}(A, r, s) \cap\left(\mathcal{T}_{i}, \mathcal{T}_{j}\right)-\operatorname{pint}(B, r, s)$ $\supseteq\left(\mathcal{T}_{i}, \mathcal{T}_{j}\right)-\operatorname{pint}(A \cap B, r, s)$.

8) $\left(\mathcal{T}_{i}, \mathcal{T}_{j}\right)-\operatorname{pint}\left(\left(\mathcal{T}_{i}, \mathcal{T}_{j}\right)-\operatorname{pint}(A, r, s), r, s\right)$ $=\left(\mathcal{T}_{i}, \mathcal{T}_{j}\right)-\operatorname{pint}(A, r, s)$. 
Theorem 2.8. Let $A$ be an intuitionistic fuzzy set in an ISBTS $\left(X, \mathcal{T}_{1}, \mathcal{T}_{2}\right)$ and $r, s \in I_{0}$. Then we have

1) $\left(\mathcal{T}_{i}, \mathcal{T}_{j}\right)-\operatorname{pint}(A, r, s)^{c}=\left(\mathcal{T}_{i}, \mathcal{T}_{j}\right)-\operatorname{pcl}\left(A^{c}, r, s\right)$.

2) $\left(\mathcal{T}_{i}, \mathcal{T}_{j}\right)-\operatorname{pcl}(A, r, s)^{c}=\left(\mathcal{T}_{i}, \mathcal{T}_{j}\right)-\operatorname{pint}\left(A^{c}, r, s\right)$.

Proof. From $\left(\mathcal{T}_{i}, \mathcal{T}_{j}\right)-\operatorname{pint}(A, r, s) \subseteq A$, we have that $A^{c} \subseteq$ $\left(\mathcal{T}_{i}, \mathcal{T}_{j}\right)-\operatorname{pint}(A, r, s)^{c}$. Since $\left(\mathcal{T}_{i}, \mathcal{T}_{j}\right)$-pint $(A, r, s)$ is IF $\left(\mathcal{T}_{i}, \mathcal{T}_{j}\right)$ $(r, s)$-preopen in $X$, the set $\left(\mathcal{T}_{i}, \mathcal{T}_{j}\right)$-pint $(A, r, s)^{c}$ is $\operatorname{IF}\left(\mathcal{T}_{i}, \mathcal{T}_{j}\right)$ $(r, s)$-preclosed. Hence,

$$
\begin{aligned}
& \left(\mathcal{T}_{i}, \mathcal{T}_{j}\right)-\operatorname{pcl}\left(A^{c}, r, s\right) \\
& \subseteq\left(\mathcal{T}_{i}, \mathcal{T}_{j}\right)-\operatorname{pcl}\left(\left(\mathcal{T}_{i}, \mathcal{T}_{j}\right)-\operatorname{pint}(A, r, s)^{c}, r, s\right) \\
& =\left(\mathcal{T}_{i}, \mathcal{T}_{j}\right)-\operatorname{pint}(A, r, s)^{c} .
\end{aligned}
$$

From the fact that $A^{c} \subseteq\left(\mathcal{T}_{i}, \mathcal{T}_{j}\right)-\operatorname{pcl}\left(A^{c}, r, s\right)$, we have $\left(\mathcal{T}_{i}, \mathcal{T}_{j}\right)-\operatorname{pcl}\left(A^{c}, r, s\right)^{c} \subseteq A$. Since $\left(\mathcal{T}_{i}, \mathcal{T}_{j}\right)-\operatorname{pcl}\left(A^{c}, r, s\right)$ is IF $\left(\mathcal{T}_{i}, \mathcal{T}_{j}\right)-(r, s)$-preclosed, $\left(\mathcal{T}_{i}, \mathcal{T}_{j}\right)-\operatorname{pcl}\left(A^{c}, r, s\right)^{c}$ is $\operatorname{IF}\left(\mathcal{T}_{i}, \mathcal{T}_{j}\right)$ $(r, s)$-preopen. Hence, we obtain that

$$
\begin{aligned}
& \left(\mathcal{T}_{i}, \mathcal{T}_{j}\right)-\operatorname{pcl}\left(A^{c}, r, s\right)^{c} \\
& =\left(\mathcal{T}_{i}, \mathcal{T}_{j}\right)-\operatorname{pint}\left(\left(\mathcal{T}_{i}, \mathcal{T}_{j}\right)-\operatorname{pcl}\left(A^{c}, r, s\right)^{c}, r, s\right) \\
& \subseteq\left(\mathcal{T}_{i}, \mathcal{T}_{j}\right)-\operatorname{pint}(A, r, s) .
\end{aligned}
$$

Thus,

$$
\left(\mathcal{T}_{i}, \mathcal{T}_{j}\right)-\operatorname{pint}(A, r, s)^{c} \subseteq\left(\mathcal{T}_{i}, \mathcal{T}_{j}\right)-\operatorname{pcl}\left(A^{c}, r, s\right) .
$$

Therefore,

$$
\left(\mathcal{T}_{i}, \mathcal{T}_{j}\right)-\operatorname{pint}(A, r, s)^{c}=\left(\mathcal{T}_{i}, \mathcal{T}_{j}\right)-\operatorname{pcl}\left(A^{c}, r, s\right) .
$$

2) Similar to 1).

\section{Intuitionistic Fuzzy Pairwise $(r, s)$ - Precontinuous Mappings}

We define the notion of intuitionistic fuzzy pairwise $(r, s)$ precontinuous mappings in intuitionistic smooth bitopological spaces, and investigate some of their characteristic properties.

Definition 3.1. Let $f:\left(X, \mathcal{T}_{1}, \mathcal{T}_{2}\right) \rightarrow\left(Y, \mathcal{U}_{1}, \mathcal{U}_{2}\right)$ be a mapping from an ISBTS $X$ to an ISBTS $Y$ and $r, s \in I_{0}$. Then $f$ is said to be IF pairwise $(r, s)$-precontinuous if $f^{-1}(A)$ is an IF $\left(\mathcal{T}_{1}, \mathcal{T}_{2}\right)-(r, s)$-preopen set in $X$ for each IF $\mathcal{U}_{1}$-r -open set $A$ in $Y$ and $f^{-1}(B)$ is an $\operatorname{IF}\left(\mathcal{T}_{2}, \mathcal{T}_{1}\right)-(s, r)$-preopen set in $X$ for each IF $\mathcal{U}_{2}$-s-open set $B$ in $Y$.
Remark 3.2. It is obvious that every IF pairwise $(r, s)$-continuous mapping is IF pairwise $(r, s)$-precontinuous. However, the following example shows that the converse need not be true.

Example 3.3. Let $\left(X, \mathcal{T}_{1}, \mathcal{T}_{2}\right)$ be an ISBTS as described in Example 2.4

Define $\mathcal{U}_{1}: I(X) \rightarrow I$ and $\mathcal{U}_{2}: I(X) \rightarrow I$ by

$$
\mathcal{U}_{1}(A)= \begin{cases}1 & \text { if } A=\underline{0}, \underline{1} \\ \frac{1}{2} & \text { if } A=A_{3} \\ 0 & \text { otherwise }\end{cases}
$$

and

$$
\mathcal{U}_{2}(A)= \begin{cases}1 & \text { if } A=\underline{0}, \underline{1} \\ 0 & \text { otherwise }\end{cases}
$$

Then $\left(\mathcal{U}_{1}, \mathcal{U}_{2}\right)$ is an ISBT on $X$. Consider a mapping $f$ : $\left(X, \mathcal{T}_{1}, \mathcal{T}_{2}\right) \rightarrow\left(X, \mathcal{U}_{1}, \mathcal{U}_{2}\right)$ defined by $f(x)=x$ and $f(y)=y$. Then $f$ is IF pairwise $\left(\frac{1}{2}, \frac{1}{3}\right)$-precontinuous. But $f$ is not an IF pairwise $\left(\frac{1}{2}, \frac{1}{3}\right)$-continuous mapping.

The following examples show that intuitionistic fuzzy pairwise $(r, s)$-semicontinuous mappings and IF pairwise $(r, s)$ precontinuous mappings are independent notions

Example 3.4. Let $\left(X, \mathcal{T}_{1}, \mathcal{T}_{2}\right)$ be an ISBTS as described in Example 2.4.

Define $\mathcal{U}_{1}: I(X) \rightarrow I$ and $\mathcal{U}_{2}: I(X) \rightarrow I$ by

$$
\mathcal{U}_{1}(A)= \begin{cases}1 & \text { if } A=\underline{0}, \underline{1} \\ 0 & \text { otherwise }\end{cases}
$$

and

$$
\mathcal{U}_{2}(A)= \begin{cases}1 & \text { if } A=\underline{0}, \underline{1} \\ \frac{1}{3} & \text { if } A=A_{5} \\ 0 & \text { otherwise }\end{cases}
$$

Then $\left(\mathcal{U}_{1}, \mathcal{U}_{2}\right)$ is an ISBT on $X$. Consider a mapping $g$ : $\left(X, \mathcal{T}_{1}, \mathcal{T}_{2}\right) \rightarrow\left(X, \mathcal{U}_{1}, \mathcal{U}_{2}\right)$ defined by $g(x)=x$ and $g(y)=y$. Then $g$ is an IF pairwise $\left(\frac{1}{2}, \frac{1}{3}\right)$-precontinuous mapping. But $g$ is not IF pairwise $\left(\frac{1}{2}, \frac{1}{3}\right)$-semicontinuous.

Example 3.5. Let $\left(X, \mathcal{T}_{1}, \mathcal{T}_{2}\right)$ be an ISBTS as described in Example 2.4.

Define $\mathcal{U}_{1}: I(X) \rightarrow I$ and $\mathcal{U}_{2}: I(X) \rightarrow I$ by

$$
\mathcal{U}_{1}(A)= \begin{cases}1 & \text { if } A=\underline{0}, \underline{1} \\ 0 & \text { otherwise }\end{cases}
$$


and

$$
\mathcal{U}_{2}(A)= \begin{cases}1 & \text { if } A=\underline{0}, \underline{1}, \\ \frac{1}{3} & \text { if } A=A_{6}, \\ 0 & \text { otherwise. }\end{cases}
$$

Then $\left(\mathcal{U}_{1}, \mathcal{U}_{2}\right)$ is an ISBT on $X$. Consider a mapping $h$ : $\left(X, \mathcal{T}_{1}, \mathcal{T}_{2}\right) \rightarrow\left(X, \mathcal{U}_{1}, \mathcal{U}_{2}\right)$ defined by $h(x)=x$ and $h(y)=y$. Then $h$ is an IF pairwise $\left(\frac{1}{2}, \frac{1}{3}\right)$-semicontinuous mapping. But $h$ is not IF pairwise $\left(\frac{1}{2}, \frac{1}{3}\right)$-precontinuous.

We can characterize the IF pairwise precontinuity by the property of inverse image of a closed set, and also by the relations of interior and closure.

Theorem 3.6. Let $f:\left(X, \mathcal{T}_{1}, \mathcal{T}_{2}\right) \rightarrow\left(Y, \mathcal{U}_{1}, \mathcal{U}_{2}\right)$ be a mapping from an ISBTS $X$ to an ISBTS $Y$ and $r, s \in I_{0}$. Then the following statements are equivalent:

1) $f$ is IF pairwise $(r, s)$-precontinuous.

2) $f^{-1}(A)$ is an $\operatorname{IF}\left(\mathcal{T}_{1}, \mathcal{T}_{2}\right)-(r, s)$-preclosed set in $X$ for each IF $\mathcal{U}_{1}$-r-closed set $A$ in $Y$ and $f^{-1}(B)$ is an IF $\left(\mathcal{T}_{2}, \mathcal{T}_{1}\right)$ - $(s, r)$-preclosed set in $X$ for each IF $\mathcal{U}_{2}$-s-closed set $B$ in $Y$.

3) For each intuitionistic fuzzy set $B$ in $Y$,

$$
\mathcal{T}_{1}-\operatorname{cl}\left(\mathcal{T}_{2}-\operatorname{int}\left(f^{-1}(B), s\right), r\right) \subseteq f^{-1}\left(\mathcal{U}_{1}-\operatorname{cl}(B, r)\right),
$$

and

$$
\mathcal{T}_{2}-\operatorname{cl}\left(\mathcal{T}_{1}-\operatorname{int}\left(f^{-1}(B), r\right), s\right) \subseteq f^{-1}\left(\mathcal{U}_{2}-\operatorname{cl}(B, s)\right)
$$

(4) For each intuitionistic fuzzy set $A$ in $X$,

$$
f\left(\mathcal{T}_{1}-\operatorname{cl}\left(\mathcal{T}_{2}-\operatorname{int}(A, s), r\right)\right) \subseteq \mathcal{U}_{1}-\operatorname{cl}(f(A), r),
$$

and

$$
f\left(\mathcal{T}_{2}-\operatorname{cl}\left(\mathcal{T}_{1}-\operatorname{int}(A, r), s\right)\right) \subseteq \mathcal{U}_{2}-\operatorname{cl}(f(A), s) .
$$

Proof. 1) $\Leftrightarrow$ 2) Trivial.

$2) \Rightarrow 3$ ) Let $B$ be an intuitionistic fuzzy set in $Y$. Then $\mathcal{U}_{1}$-cl $(B, r)$ is IF $\mathcal{U}_{1}$ - $r$-closed and $\mathcal{U}_{2}-\operatorname{cl}(B, s)$ is IF $\mathcal{U}_{2}$-s-closed in $Y$. Thus, by 2$), f^{-1}\left(\mathcal{U}_{1}-\operatorname{cl}(B, r)\right)$ is an $\operatorname{IF}\left(\mathcal{T}_{1}, \mathcal{T}_{2}\right)-(r, s)$ preclosed set and $f^{-1}\left(\mathcal{U}_{2}-\operatorname{cl}(B, s)\right)$ is an $\operatorname{IF}\left(\mathcal{T}_{2}, \mathcal{T}_{1}\right)-(s, r)$ preclosed set in $X$. Hence, we have

$$
\mathcal{T}_{1}-\operatorname{cl}\left(\mathcal{T}_{2}-\operatorname{int}\left(f^{-1}(B), s\right), r\right)
$$

$$
\begin{aligned}
& \subseteq \mathcal{T}_{1}-\operatorname{cl}\left(\mathcal{T}_{2}-\operatorname{int}\left(f^{-1}\left(\mathcal{U}_{1}-\operatorname{cl}(B, r)\right), s\right), r\right) \\
& \subseteq f^{-1}\left(\mathcal{U}_{1}-\operatorname{cl}(B, r)\right)
\end{aligned}
$$

and

$$
\begin{aligned}
& \mathcal{T}_{2}-\operatorname{cl}\left(\mathcal{T}_{1}-\operatorname{int}\left(f^{-1}(B), r\right), s\right) \\
& \subseteq \mathcal{T}_{2}-\operatorname{cl}\left(\mathcal{T}_{1}-\operatorname{int}\left(f^{-1}\left(\mathcal{U}_{2}-\operatorname{cl}(B, s)\right), r\right), s\right) \\
& \subseteq f^{-1}\left(\mathcal{U}_{2}-\operatorname{cl}(B, s)\right) .
\end{aligned}
$$

$3) \Rightarrow 4$ ) Let $A$ be an intuitionistic fuzzy set in $X$. Then by 3), we obtain

$$
\begin{aligned}
\mathcal{T}_{1}-\operatorname{cl}\left(\mathcal{T}_{2}-\operatorname{int}(A, s), r\right) & \subseteq \mathcal{T}_{1}-\operatorname{cl}\left(\mathcal{T}_{2}-\operatorname{int}\left(f^{-1}(f(A)), s\right), r\right) \\
& \subseteq f^{-1}\left(\mathcal{U}_{1}-\operatorname{cl}(f(A), r)\right),
\end{aligned}
$$

and

$$
\begin{aligned}
\mathcal{T}_{2}-\operatorname{cl}\left(\mathcal{T}_{1}-\operatorname{int}(A, r), s\right) & \subseteq \mathcal{T}_{2}-\operatorname{cl}\left(\mathcal{T}_{1}-\operatorname{int}\left(f^{-1}(f(A)), r\right), s\right) \\
& \subseteq f^{-1}\left(\mathcal{U}_{2}-\operatorname{cl}(f(A), s)\right) .
\end{aligned}
$$

Hence

$$
f\left(\mathcal{T}_{1}-\operatorname{cl}\left(\mathcal{T}_{2}-\operatorname{int}(A, s), r\right)\right) \subseteq \mathcal{U}_{1}-\operatorname{cl}(f(A), r),
$$

and

$$
f\left(\mathcal{T}_{2}-\operatorname{cl}\left(\mathcal{T}_{1}-\operatorname{int}(A, r), s\right)\right) \subseteq \mathcal{U}_{2}-\operatorname{cl}(f(A), s) .
$$

4) $\Rightarrow 2$ ) Let $A$ be any IF $\mathcal{U}_{1}-r$-closed set and $B$ any IF $\mathcal{U}_{2}-s$ closed set in $Y$. By (4), we have

$$
\begin{aligned}
f\left(\mathcal{T}_{1}-\operatorname{cl}\left(\mathcal{T}_{2}-\operatorname{int}\left(f^{-1}(A), s\right), r\right)\right) & \subseteq \mathcal{U}_{1}-\operatorname{cl}\left(f\left(f^{-1}(A)\right), r\right) \\
& \subseteq \mathcal{U}_{1}-\operatorname{cl}(A, r)=A,
\end{aligned}
$$

and

$$
\begin{aligned}
f\left(\mathcal{T}_{2}-\operatorname{cl}\left(\mathcal{T}_{1}-\operatorname{int}\left(f^{-1}(B), r\right), s\right)\right) & \subseteq \mathcal{U}_{2}-\operatorname{cl}\left(f\left(f^{-1}(B)\right), s\right) \\
& \subseteq \mathcal{U}_{2}-\operatorname{cl}(B, s)=B .
\end{aligned}
$$

Hence

$$
\mathcal{T}_{1}-\operatorname{cl}\left(\mathcal{T}_{2}-\operatorname{int}\left(f^{-1}(A), s\right), r\right) \subseteq f^{-1}(A),
$$

and

$$
\mathcal{T}_{2}-\operatorname{cl}\left(\mathcal{T}_{1}-\operatorname{int}\left(f^{-1}(B), r\right), s\right) \subseteq f^{-1}(B) .
$$

Therefore, $f^{-1}(A)$ is an IF $\left(\mathcal{T}_{1}, \mathcal{T}_{2}\right)-(r, s)$-preclosed set and $f^{-1}(B)$ is an IF $\left(\mathcal{T}_{2}, \mathcal{T}_{1}\right)-(s, r)$-preclosed set in $X$. 
We can characterize the IF pairwise precontinuity by the relations of preinterior or preclosure.

Theorem 3.7. Let $f:\left(X, \mathcal{T}_{1}, \mathcal{T}_{2}\right) \rightarrow\left(Y, \mathcal{U}_{1}, \mathcal{U}_{2}\right)$ be a mapping from an ISBTS $X$ to an ISBTS $Y$ and $r, s \in I_{0}$. Then the following statements are equivalent:

1) $f$ is IF pairwise $(r, s)$-precontinuous.

2) For each intuitionistic fuzzy set $A$ in $X$,

$$
f\left(\left(\mathcal{T}_{1}, \mathcal{T}_{2}\right)-\operatorname{pcl}(A, r, s)\right) \subseteq \mathcal{U}_{1}-\operatorname{cl}(f(A), r),
$$

and

$$
f\left(\left(\mathcal{T}_{2}, \mathcal{T}_{1}\right)-\operatorname{pcl}(A, s, r)\right) \subseteq \mathcal{U}_{2}-\operatorname{cl}(f(A), r)
$$

(3) For each intuitionistic fuzzy set $B$ in $Y$,

$$
\left(\mathcal{T}_{1}, \mathcal{T}_{2}\right)-\operatorname{pcl}\left(f^{-1}(B), r, s\right) \subseteq f^{-1}\left(\mathcal{U}_{1}-\operatorname{cl}(B, r)\right)
$$

and

$$
\left(\mathcal{T}_{2}, \mathcal{T}_{1}\right)-\operatorname{pcl}\left(f^{-1}(B), s, r\right) \subseteq f^{-1}\left(\mathcal{U}_{2}-\operatorname{cl}(B, s)\right)
$$

(4) For each intuitionistic fuzzy set $B$ in $Y$,

$$
f^{-1}\left(\mathcal{U}_{1}-\operatorname{int}(B, r)\right) \subseteq\left(\mathcal{T}_{1}, \mathcal{T}_{2}\right)-\operatorname{pint}\left(f^{-1}(B), r, s\right)
$$

and

$$
f^{-1}\left(\mathcal{U}_{2}-\operatorname{int}(B, s)\right) \subseteq\left(\mathcal{T}_{2}, \mathcal{T}_{1}\right)-\operatorname{pint}\left(f^{-1}(B), s, r\right)
$$

Proof. 1) $\Rightarrow 2$ ) Let $A$ be an intuitionistic fuzzy set in $X$. Then $\mathcal{U}_{1}-\operatorname{cl}(f(A), r)$ is $\operatorname{IF} \mathcal{U}_{1}-r$-closed and $\mathcal{U}_{2}-\operatorname{cl}(f(A), s)$ is $\operatorname{IF} \mathcal{U}_{2}-$ $s$-closed in $Y$. Since $f$ is IF pairwise $(r, s)$-precontinuous, $f^{-1}\left(\mathcal{U}_{1}-\operatorname{cl}(f(A), r)\right)$ is an IF $\left(\mathcal{T}_{1}, \mathcal{T}_{2}\right)-(r, s)$-preclosed set and $f^{-1}\left(\mathcal{U}_{2}-\operatorname{cl}(f(A), s)\right)$ is an IF $\left(\mathcal{T}_{2}, \mathcal{T}_{1}\right)-(s, r)$-preclosed set in $X$. Thus

$$
\begin{aligned}
& \left(\mathcal{T}_{1}, \mathcal{T}_{2}\right)-\operatorname{pcl}(A, r, s) \\
& \subseteq\left(\mathcal{T}_{1}, \mathcal{T}_{2}\right)-\operatorname{pcl}\left(f^{-1}\left(\mathcal{U}_{1}-\operatorname{cl}(f(A), r)\right), r, s\right) \\
& =f^{-1}\left(\mathcal{U}_{1}-\operatorname{cl}(f(A), r)\right),
\end{aligned}
$$

and

$$
\begin{aligned}
& \left(\mathcal{T}_{2}, \mathcal{T}_{1}\right)-\operatorname{pcl}(A, s, r) \\
& \subseteq\left(\mathcal{T}_{2}, \mathcal{T}_{1}\right)-\operatorname{pcl}\left(f^{-1}\left(\mathcal{U}_{2}-\operatorname{cl}(f(A), s)\right), s, r\right) \\
& =f^{-1}\left(\mathcal{U}_{2}-\operatorname{cl}(f(A), s)\right) .
\end{aligned}
$$

Therefore

$$
f\left(\left(\mathcal{T}_{1}, \mathcal{T}_{2}\right)-\operatorname{pcl}(A, r, s)\right) \subseteq \mathcal{U}_{1}-\operatorname{cl}(f(A), r),
$$

and

$$
f\left(\left(\mathcal{T}_{2}, \mathcal{T}_{1}\right)-\operatorname{pcl}(A, s, r)\right) \subseteq \mathcal{U}_{2}-\operatorname{cl}(f(A), s)
$$

(2) $\Rightarrow$ (3) Let $B$ be an intuitionistic fuzzy set in $Y$. Then by (2), we have

$$
\begin{aligned}
f\left(\left(\mathcal{T}_{1}, \mathcal{T}_{2}\right)-\operatorname{pcl}\left(f^{-1}(B), r, s\right)\right) & \subseteq \mathcal{U}_{1}-\operatorname{cl}\left(f\left(f^{-1}(B)\right), r\right) \\
& \subseteq \mathcal{U}_{1}-\operatorname{cl}(B, r)
\end{aligned}
$$

and

$$
\begin{aligned}
f\left(\left(\mathcal{T}_{2}, \mathcal{T}_{1}\right)-\operatorname{pcl}\left(f^{-1}(B), s, r\right)\right) & \subseteq \mathcal{U}_{2}-\operatorname{cl}\left(f\left(f^{-1}(B)\right), s\right) \\
& \subseteq \mathcal{U}_{2}-\operatorname{cl}(B, s)
\end{aligned}
$$

Hence

$$
\left(\mathcal{T}_{1}, \mathcal{T}_{2}\right)-\operatorname{pcl}\left(f^{-1}(B), r, s\right) \subseteq f^{-1}\left(\mathcal{U}_{1}-\operatorname{cl}(B, r)\right)
$$

and

$$
\left(\mathcal{T}_{2}, \mathcal{T}_{1}\right)-\operatorname{pcl}\left(f^{-1}(B), s, r\right) \subseteq f^{-1}\left(\mathcal{U}_{2}-\operatorname{cl}(B, s)\right)
$$

(3) $\Rightarrow(4)$ Let $B$ be an intuitionistic fuzzy set in $Y$. Then by (3), we have

$$
\left(\mathcal{T}_{1}, \mathcal{T}_{2}\right)-\operatorname{pcl}\left(f^{-1}\left(B^{c}\right), r, s\right) \subseteq f^{-1}\left(\mathcal{U}_{1}-\operatorname{cl}\left(B^{c}, r\right)\right)
$$

and

$$
\left(\mathcal{T}_{2}, \mathcal{T}_{1}\right)-\operatorname{pcl}\left(f^{-1}\left(B^{c}\right), s, r\right) \subseteq f^{-1}\left(\mathcal{U}_{2}-\operatorname{cl}\left(B^{c}, s\right)\right)
$$

Hence

$$
\begin{aligned}
f^{-1}\left(\mathcal{U}_{1}-\operatorname{int}(B, r)\right) & =\left(f^{-1}\left(\mathcal{U}_{1}-\operatorname{cl}\left(B^{c}, r\right)\right)\right)^{c} \\
& \subseteq\left(\mathcal{T}_{1}, \mathcal{T}_{2}\right)-\operatorname{pcl}\left(f^{-1}\left(B^{c}\right), r, s\right)^{c} \\
& =\left(\mathcal{T}_{1}, \mathcal{T}_{2}\right)-\operatorname{pint}\left(f^{-1}(B), r, s\right),
\end{aligned}
$$

and

$$
\begin{aligned}
f^{-1}\left(\mathcal{U}_{2}-\operatorname{int}(B, s)\right) & =\left(f^{-1}\left(\mathcal{U}_{2}-\operatorname{cl}\left(B^{c}, s\right)\right)\right)^{c} \\
& \subseteq\left(\mathcal{T}_{2}, \mathcal{T}_{1}\right)-\operatorname{pcl}\left(f^{-1}\left(B^{c}\right), s, r\right)^{c} \\
& =\left(\mathcal{T}_{2}, \mathcal{T}_{1}\right)-\operatorname{pint}\left(f^{-1}(B), s, r\right) .
\end{aligned}
$$

$4) \Rightarrow 1$ ) Let $A$ be any IF $\mathcal{U}_{1}-r$-open set and $B$ any IF $\mathcal{U}_{2}-s$ - 
open set in $Y$. Then $\mathcal{U}_{1}$-int $(A, r)=A$ and $\mathcal{U}_{2}$-int $(B, s)=B$. Hence

$$
\begin{aligned}
f^{-1}(A) & =f^{-1}\left(\mathcal{U}_{1}-\operatorname{int}(A, r)\right) \\
& \subseteq\left(\mathcal{T}_{1}, \mathcal{T}_{2}\right)-\operatorname{pint}\left(f^{-1}(A), r, s\right) \\
& \subseteq f^{-1}(A),
\end{aligned}
$$

and

$$
\begin{aligned}
f^{-1}(B) & =f^{-1}\left(\mathcal{U}_{2}-\operatorname{int}(B, s)\right) \\
& \subseteq\left(\mathcal{T}_{2}, \mathcal{T}_{1}\right)-\operatorname{pint}\left(f^{-1}(B), s, r\right) \\
& \subseteq f^{-1}(B) .
\end{aligned}
$$

Hence

$$
f^{-1}(A)=\left(\mathcal{T}_{1}, \mathcal{T}_{2}\right)-\operatorname{pint}\left(f^{-1}(A), r, s\right),
$$

and

$$
f^{-1}(B)=\left(\mathcal{T}_{2}, \mathcal{T}_{1}\right)-\operatorname{pint}\left(f^{-1}(B), s, r\right) .
$$

Thus, $f^{-1}(A)$ is an $\operatorname{IF}\left(\mathcal{T}_{1}, \mathcal{T}_{2}\right)-(r, s)$-preopen set and $f^{-1}(B)$ is an IF $\left(\mathcal{T}_{2}, \mathcal{T}_{1}\right)-(s, r)$-preopen set in $X$. Therefore, $f$ is IF pairwise $(r, s)$-precontinuous.

Theorem 3.8. Let $f:\left(X, \mathcal{T}_{1}, \mathcal{T}_{2}\right) \rightarrow\left(Y, \mathcal{U}_{1}, \mathcal{U}_{2}\right)$ be a bijective mapping from an ISBTS $X$ to an ISBTS $Y$ and $r, s \in I_{0}$. Then $f$ is IF pairwise $(r, s)$-precontinuous if and only if

$$
\mathcal{U}_{1}-\operatorname{int}(f(A), r) \subseteq f\left(\left(\mathcal{T}_{1}, \mathcal{T}_{2}\right)-\operatorname{pint}(A, r, s)\right),
$$

and

$$
\mathcal{U}_{2}-\operatorname{int}(f(A), s) \subseteq f\left(\left(\mathcal{T}_{2}, \mathcal{T}_{1}\right)-\operatorname{pint}(A, s, r)\right)
$$

for each intuitionistic fuzzy set $A$ in $X$.

Proof. Let $A$ be an intuitionistic fuzzy set in $X$. Since $f$ is one-to-one, by Theorem 3.7, we obtain

$$
\begin{aligned}
f^{-1}\left(\mathcal{U}_{1}-\operatorname{int}(f(A), r)\right) & \subseteq\left(\mathcal{T}_{1}, \mathcal{T}_{2}\right)-\operatorname{pint}\left(f^{-1}(f(A)), r, s\right) \\
& =\left(\mathcal{T}_{1}, \mathcal{T}_{2}\right)-\operatorname{pint}(A, r, s),
\end{aligned}
$$

and

$$
\begin{aligned}
f^{-1}\left(\mathcal{U}_{2}-\operatorname{int}(f(A), s)\right) & \subseteq\left(\mathcal{T}_{2}, \mathcal{T}_{1}\right)-\operatorname{pint}\left(f^{-1}(f(A)), s, r\right) \\
& =\left(\mathcal{T}_{2}, \mathcal{T}_{1}\right)-\operatorname{pint}(A, s, r) .
\end{aligned}
$$

Since $f$ is onto, we have

$$
\mathcal{U}_{1}-\operatorname{int}(f(A), r)=f\left(f^{-1}\left(\mathcal{U}_{1}-\operatorname{int}(f(A), r)\right)\right)
$$

$$
\subseteq f\left(\left(\mathcal{T}_{1}, \mathcal{T}_{2}\right)-\operatorname{pint}(A, r, s)\right)
$$

and

$$
\begin{aligned}
\mathcal{U}_{2}-\operatorname{int}(f(A), s) & =f\left(f^{-1}\left(\mathcal{U}_{2}-\operatorname{int}(f(A), s)\right)\right) \\
& \subseteq f\left(\left(\mathcal{T}_{2}, \mathcal{T}_{1}\right)-\operatorname{pint}(A, s, r)\right) .
\end{aligned}
$$

Conversely, let $B$ be an intuitionistic fuzzy set in $Y$. Since $f$ is onto, we have

$$
\begin{aligned}
\mathcal{U}_{1}-\operatorname{int}(B, r) & =\mathcal{U}_{1}-\operatorname{int}\left(f\left(f^{-1}(B)\right), r\right) \\
& \subseteq f\left(\left(\mathcal{T}_{1}, \mathcal{T}_{2}\right)-\operatorname{pint}\left(f^{-1}(B), r, s\right),\right.
\end{aligned}
$$

and

$$
\begin{aligned}
\mathcal{U}_{2}-\operatorname{int}(B, s) & =\mathcal{U}_{2}-\operatorname{int}\left(f\left(f^{-1}(B)\right), s\right) \\
& \subseteq f\left(\left(\mathcal{T}_{2}, \mathcal{T}_{1}\right)-\operatorname{pint}\left(f^{-1}(B), s, r\right) .\right.
\end{aligned}
$$

Since $f$ is one-to-one, we obtain

$$
\begin{aligned}
f^{-1}\left(\mathcal{U}_{1}-\operatorname{int}(B, r)\right) & \subseteq f^{-1}\left(f\left(\left(\mathcal{T}_{1}, \mathcal{T}_{2}\right)-\operatorname{pint}\left(f^{-1}(B), r, s\right)\right)\right) \\
& =\left(\mathcal{T}_{1}, \mathcal{T}_{2}\right)-\operatorname{pint}\left(f^{-1}(B), r, s\right),
\end{aligned}
$$

and

$$
\begin{aligned}
f^{-1}\left(\mathcal{U}_{2}-\operatorname{int}(B, s)\right) & \subseteq f^{-1}\left(f\left(\left(\mathcal{T}_{2}, \mathcal{T}_{1}\right)-\operatorname{pint}\left(f^{-1}(B), s, r\right)\right)\right) \\
& =\left(\mathcal{T}_{2}, \mathcal{T}_{1}\right)-\operatorname{pint}\left(f^{-1}(B), s, r\right) .
\end{aligned}
$$

Therefore, by Theorem 3.7, $f$ is an IF pairwise $(r, s)$-precontinuous mapping.

\section{Conclusions}

In this paper, we introduced a new notion of intuitionistic fuzzy pairwise precontinuous mappings in intuitionistic smooth bitopological spaces. By investigating the relations of closedness, interior, closure, preinterior, and preclosure, we obtained six equivalent conditions for intuitionistic fuzzy pairwise precontinuous mappings in Theorem 3.6 and Theorem 3.7. Furthermore, we obtained another equivalent condition for a bijective mapping in Theorem 3.8.

\section{Acknowledgments}

This work was conducted during the research year of Chungbuk National University in 2018. 


\section{References}

[1] C. L. Chang, "Fuzzy topological spaces,” Journal of Mathematical Analysis and Applications, vol. 24, no. 1, 182190, 1968.

[2] A. P. Sostak, “On a fuzzy topological structure," in Proceedings of the 13th Winter School on Abstract Analysis, Srni, Czech Republic, 1985, pp. 89-103.

[3] A. P. Sostak, "Two decades of fuzzy topology: basic ideas, notions, and results," Russian Mathematical Surveys, vol. 44, no. 6, pp. 125-186, 1989.

[4] A. A. Ramadan, "Smooth topological spaces," Fuzzy Sets and Systems, vol. 48, no. 3, pp. 371-375, 1992. https: //doi.org/10.1016/0165-0114(92)90352-5

[5] A. Kandil, "Biproximities and fuzzy bitopological spaces," Simon Stevin, vol. 63, pp. 45-66, 1989.

[6] E. P. Lee, "Pairwise semicontinuous mapping in smooth bitopological spaces," Journal of Korean Institute of Intelligent Systems, vol. 12, no. 3, pp. 269-274, 2002. https://doi.org/10.5391/JKIIS.2002.12.3.269

[7] E. P. Lee, Y. B. Im, and H. Han, "Semiopen sets on smooth bitopological spaces," Far East Journal of Mathematical Sciences, vol. 3, no. 3, pp. 493-511, 2001.

[8] D. Coker, "An introduction to intuitionistic fuzzy topological spaces," Fuzzy Sets and systems, vol. 88, no. 1, pp. 8189, 1997. https://doi.org/10.1016/S0165-0114(96)000760

[9] D. Coker and M. Demirci, "An introduction to intuitionistic fuzzy topological spaces in Sostak's sense," Busefal, vol. 67, pp. 67-76, 1996.

[10] K. T. Atanassov, "Intuitionistic fuzzy sets," Fuzzy Sets and systems, vol. 20, no. 1, pp. 87-96, 1986. https://doi. org/10.1016/S0165-0114(86)80034-3
[11] T. K. Mondal and S. K. Samanta, "On intuitionistic gradation of openness," Fuzzy Sets and Systems, vol. 131, no. 3, pp. 323-336, 2002. https://doi.org/10.1016/S01650114(01)00235-4

[12] P. K. Lim, S. R. Kim, and K. Hur, "Intuitionistic smooth topological spaces," Journal of Korean Institute of Intelligent Systems, vol. 20, no. 6, pp. 875-883. 2010. https://doi.org/10.5391/JKIIS.2010.20.6.875

[13] J. T. Kim and S. J. Lee, "Intuitionistic smooth bitopological spaces and continuity," International Journal of Fuzzy Logic and Intelligent Systems, vol. 14, no. 1, pp. 49-56, 2014. https://doi.org/10.5391/IJFIS.2014.14.1.49

[14] J. T. Kim and S. J. Lee, "Pairwise semiopen and semiclosed mappings in intuitionistic smooth bitopological spaces," Journal of the Chungcheong Mathematical Society, vol. 29, no. 2, pp. 367-374, 2016. https://doi.org/10. 14403/jcms.2016.29.2.367

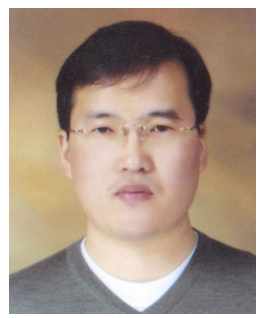

Jin Tae Kim received his $\mathrm{Ph}$. D. degree from Chungbuk National University in 2012. His research interests include general topology and fuzzy topology. He is a member of KIIS and KMS.

E-mail: kjtmath@hanmail.net

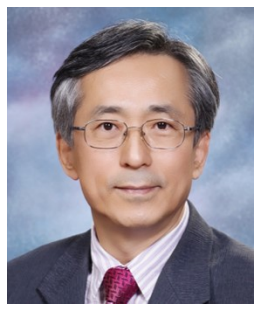

Seok Jong Lee received his M. S. and Ph. D. degrees from Yonsei University in 1986 and 1990, respectively. He is a professor at the Department of Mathematics, Chungbuk National University since 1989 . He was a visiting scholar in Carleton University from 1995 to 1996, and Wayne State University from 2003 to 2004. His research interests include general topology and fuzzy topology. He is a member of KIIS and KMS.

E-mail: sj1@cbnu.ac.kr 